\title{
The Other Side of Neurotechnologies
}

\author{
Kristian Elersic \\ University of Ljubljana \\ Faculty of Arts \\ Ljubljana, Slovenia \\ ekristian238@gmail.com
}

\begin{abstract}
As a response to the overly-optimistic view of technology, I will present some of the obstacles when using new neurotechnologies, specifically cognitive enhancers. Those obstacles come in a form of physical limitations, such as not being able to develop methods sophisticated enough that they would have no (or minimal) negative side-effects, and in a form of ethical limitations, since even if these new technologies were physically possible, the extent to which we should use these technologies is not fully explored. To fully consider such obstacles, I propose an outline of the ethical framework that should be used when thinking of applying new, future or even old cognitive technologies. Furthermore, I pose some questions for further analysis of the intertwining of technoscientific progress with social dynamics.
\end{abstract}

Keywords: Neurotechnology, Transhumanism, Enhancement, Cognition, Ethics

Received: 28 September 2018, Revised 14 December 2018, Accepted 15 January 2019

DOI: $10.6025 / \mathrm{jic} / 2019 / 10 / 2 / 55-59$

\section{(C) 2019 DLINE. All Rights Reserved}

\section{Introduction}

Neurotechnology, technology that has a fundamental impact on how we understand the brain, cognitive processes and consciousness, is rapidly developing. Some of the examples of neurotechnologies are: imaging technology such as magnetic resonance imaging (MRI), electroencephalogram (EEG) or positron emission tomography (PET), brain stimulations such as transcranial magnetic stimulations (TMS) or transcranial direct current stimulation (tDCS), implant technologies, pharmaceuticals, gene editing, cell therapy and brain-computer interfaces. In this article I will be focusing on technologies that aim to improve the cognitive processes - cognitive enhancers. There is not a specific technology that suits this purpose only. It is rather a combination of different technologies that are believed to alter cognitive processes: gene editing, pharmaceuticals and brain stimulations.

My whole stance is formed as a response to the overly-optimistic view of technology - which is that technology can save many of our problems, if not all of them. At no point in this paper will I go into detailed discussion of any topic, but will rather open a broad field of questions that are important to consider when analysing neurotechnology and human enhancement. In the first section of this paper, I will describe what I mean with the term "overlyoptimistic view of technology". In the second section, I will describe shorty what are the cognitive enhancers. Then I will continue by presenting some of the physical limitations of the 
enhancers, namely the limitations for gene editing, pharmaceuticals and brain stimulations. Then I will set the ethical framework that will serve the purpose of considering or questioning any intervention. Finally, I will try to think of the possible connections of the techno-scientific progress with society.

\section{Overly-optimistic View of Technology and Enhancement}

As a student of science, I am mainly surrounded by scientific literature and scientists. What I have found is that there is a general

lack of interest in interdisciplinary discussion of impacts that techno-scientific progress has on a society. It almost seems that there prevails an opinion of that technology and science cannot be misused - over-optimism or maybe even ignorance. To clarify

further, this opinion comes from my student surroundings - University of Ljubljana, Biotechnical faculty. I haven't done any analysis of other universities. Of course, this interdisciplinary topic is not strictly a scientific one, but nonetheless it is important.

Therefore, I think that discussions of this kind should be included in the scientific community, especially when it comes to biotechnology that directly addresses and modifies the human condition. On the other hand, beside lack of interest of my academic surroundings, there is also an emerging movement that is overlyoptimistic about technology. It is called transhumanism. Max More, philosopher and futurist, defined it:"

“... [as] both a reason-based philosophy and a cultural movement that affirms the possibility and desirability of funda mentally improving the human condition by means of science and technology. Transhumanists seek the continuation and acceleration of the evolution of intelligent life beyond its currently human form and human limitations by means of science and technology, guided by life-promoting principles and values." [1]

A few of the common characteristics of the mentioned philosophy are: a) optimistic view of progress of science and technology, b) aiming at the advancement of humans - to alleviate our biological constrains so that we could live longer and healthier, c) using science and technology as the main means of advancement and d) claiming that enhancement will transform the whole meaning of being human. In other words, they state that technology will help us as a humanity to overcome our current problems, it will help us to be more ethical, healthy, well in both economic and ecologic sense. Transhumanists also believe that by doing so we will transform ourselves into some future form where we will no longer be able to state we are the same as we were before as species - we will become post-human in a transhumanist sense.

For this purpose I want to analyse and present some questions related to the human bio-enhancement, specifically cognitive enhancement. My aim is not to discredit latest scientific development. New knowledge and new technologies are appreciated but with them comes a responsibility of how and when to use them. My aim is to stimulate an interdisciplinary discourse of the mentioned subject - human enhancement and neurotechnologies. In other words, I want that we take a closer look at the other, darker side of new neurotechnologies that aim at enhancing humans, because the bright side is known to well.

\section{Cognitive Enhancers}

Cognitive enhancers are technologies that enhance cognitive processes. Like doping in sports that enhances physical performance, cognitive enhancers stimulate memory, perception, concentration, motivation, learning, problem solving and computation. [2 3] Most widely known are the so-called "smart drugs" - cognitive enhancers in the form of pharmaceuticals (substances). Those are already used for medicinal and enhancing purposes. [3 4] Besides that, scientists are developing methods for cognitive enhancement in the form of genetic engineering and brain stimulations. In a broader sense, even traditional cognitive training can be classified as one form of cognitive enhancers, but I will rather focus on technological enhancement. ${ }^{1}$

\section{Physical Limitations}

When describing neurotechnological interventions for enhancement purposes, we have mentioned three types of modifica-

${ }^{1}$ A special kind of cognitive enhancers are moral enhancers that are more strictly concerned with improving our moral behaviour. [5]

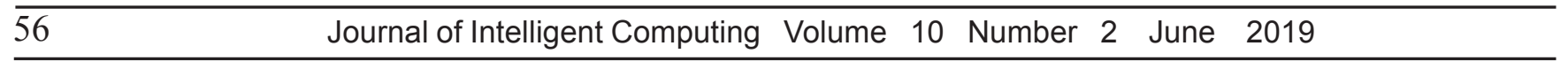


tions: a) genetic engineering, b) drug intake and c) brain stimulation. In this section I will present some of the (possible) limitations of mentioned technologies.

\subsection{Genetic Engineering}

Genetic engineering started in 1973 and has very quickly spread onto many fields of industry and research. In our case, genetic engineering would be used for manipulation of certain cognitive trait like attention, IQ, memory and so on. For example, we could genetically modify embryo's DNA, or later in life insert some gene products to tissues or organs (RNA or protein insertions), or "knock out", that is, make inoperative, some genes (gene knockout with DNA cutting proteins), or we could select one out of many embryos with the most preferable genome (prenatal diagnostic or embryo selection). But when it comes to genetics, we often misinterpret that there is a direct link between a gene and a trait. We have to bear in mind that only a few traits are monogenetic, meaning that there is only one gene that influences a certain trait. In most cases, there are multiple genes that affect one trait. Besides that, in many cases one gene also affects many traits. [6] Due to this phenomenon (complex genetic traits) it is very hard to predict the total outcome of such genetic manipulation. This is nicely illustrated by the study when researchers improved mouse's memory and simultaneously increases its sensitivity to pain. [7 8 9]

\subsection{Pharmaceuticals}

Enhanced cognition can be also achieved with numerous substances, even with dietary supplements, such as caffeine and nicotine, but for our purpose I will describe pharmaceuticals (which are classified as technological interventions). One of such pharmaceuticals is methylphenidate, commercially known as Ritalin. Ritalin is nowadays widely used not just for treating the attention deficit hyperactivity disorder (ADHD), but is popular as a cognitive enhancer among students/academics with the purpose of achieving better study or academical results. [3 4] Other similar substances are: amphetamines, modafinil, atomoxetine, reboxetine, donepezil, galatamine, rivastigmine and memantine. When prescribing these drugs it is important to take into account obstacles that may come with the use of these substances. One such obstacle is that substances often have side effects such as nausea, vomiting or cramps. The second obstacle is that brain responds to the chemicals in such a way that a too high or too low concentration of substance might not produce optimal brain functioning effects. ${ }^{2}$ In some individuals one dose may cause a positive effect while in the others the effect would be negative. [3] The third obstacle is that a drug may positively affect one brain function while simultaneously negatively affect the other. ${ }^{3}$ Last but not least, it is important to add that we lack long term studies that would tell us how safe is the use of these drugs - in other words, what are the long-term side effects of such enhancers. [3 $1011]$

\subsection{Brain Stimulation}

Brain stimulation is a technology using electrical currents or magnetic field (which induces electric currents) to stimulate certain parts of brain tissue. Methods of brain simulation can be further divided into invasive and non-invasive ones. The invasive method requires application of currents directly to the brain's surface or brain's core. In order to achieve this, we have to be invasive - the skull has to be opened and sometimes even the brain must be cut. Using the non-invasive methods, the currents or magnetic fields are applied over the head's surface. This is called non-invasive brain stimulation (NBS), examples of which are transcranial magnetic stimulation (TMS) and transcranial direct current stimulation (tDCS). For our further discussion it is not of a great importance what kind of technique is used.

Opposition to the overly optimistic view towards this form of cognitive enhancement may come from understanding brain's functioning. Key concept in this opposition is net zero-sum framework proposed by Brem et al. (2014). [12] Authors state that this concept is grounded on the physical principle of conservation of energy in a closed system. Furthermore, they clarify that, in this notion, brain operates within the constraints of a finite amount of energy and processing power. Not meaning that all the brains and the same brain through the life span do have equal amount of energy and processing power, but that an x-amount of energy and processing power is distributed throughout the brain at any given time. When demands shift, so does the distribution of energy and processing power. Most importantly, total sum of brain's activity stays the same. In other words, brain operates in net zero-sum framework. If this prediction is correct, NBS would enhance some brain function, but at the same time degrade other (compensation/cost). Some evidence accounting for this hypothesis comes from the phenomena of paradoxical functional

${ }^{2}$ This applies for future drugs too. This molecular dynamics is called U-shaped relationship between substance and receptor. [3]

${ }^{3}$ Example: when rigastimin improves learning on a motor task and making associations between symbols and digits, but can at the same time impair verbal and visual episodic memory. [3 10] 
facilitation. [12 13] This phenomenon appears when damage to an intact area of the brain normalizes a previously reduced level of functioning (compensation for previous loss - re-establishing balance between functional brain networks) or when a patient with damage performs a certain task better than before or better than healthy control subjects (energy and processing power of damaged brain tissue was distributed to other brain parts that are now enhanced). Authors conclude with raising bioethical concerns: "Is it acceptable to improve certain brain functions at the cost of others and can we take the responsibility for its impact on the individual and on society?" (p.14-15) They also state that "current neuroenhancment studies emphasize positive outcome of specific functions and concentrate on individual improvements, while related topics such as risk and safety, as well as social and moral factors are neglected or restricted to specific inquiries.” (p.15) [12]

\section{Ethical Framework and Intertwining of Technoscientific Progress with Society}

In the previous section I have described some physical limitations that we may encounter when applying neuroenhancing technologies. Now I want to state that even if we do develop better technology, we still have to consider questions from a very broad spectrum of topics.

Firstly, I want to propose an ethical framework consisting of three points that we have to consider in a discussions about any human interventions such as bio-enhancing technologies. (1) First is about personal freedom. (2) Second is about the line between long-term sustainability and short-term solutions. And (3) third is about ecological integrity/sustainability. For example: 1) do enhancers that aim to limit our immoral behaviours, limit our personal freedom? Or, do cognitive enhancers prescribed to children for enhancement of a specific trait, limit their freedom to choose/develop other characteristic that they would love to have? Or 2) are costs and side-effects of bio-enhancing accounted for? Are those short term solutions sustainable or do they just prolong the problem or maybe even worsen the situation? Where is the boundary between enhancement and a disease - former without the need (but with the want), latter with the immediate need? And 3) is this technology ecologically sustainable? Does it produce a nonsustainable mentality - that which is not able to think in a nonselfish way or in an ecological way or in terms of long-term solutions? What will this technology do to overpopulation, species extinction, how many waste will it produce? Those are just a few questions stated to further clarify the mentioned ethical framework.

Secondly, I want to briefly mention how broad the field of investigation in this and similar topics should be. Nature of questioning and regulating techno-scientific progress, especially when we think of interfering with our nature (enhancement), exceeds fields of natural sciences. It includes (or should include) questions from philosophy of science (what is knowledge, what is science, its place and role in modern and future society), philosophy of progress (genealogy of progress, what is progress), intertwining of the techno-scientific progress and the economic system (how is modern free market system influencing technoscientific progress, business of science), intertwining of the market and the values/ideological system (how does the market with advertising influence the values and how do these values afterwards influence the market) and the application of technology in political systems (possibility of new technologies for achieving political goals, possibility of manipulation and control). Furthermore, we have to ask ourselves why do we want enhancement: is it a by-product of economic system (competition - to be better and to get a better job), or is it a by-product of modern values (for example, value of ideal life - long and healthy with no negativity), or a product of advertisement or political viewpoint. We ought to analyse all of the mentioned subfields before interfering with human nature with the use of enhancers to the extent that would limit our capacity undo the damage.

\section{Conclusion}

Development of new technologies opens up many questions - from physical limitations to ethical considerations. I have focused on neurotechnologies, specifically cognitive enhancers. My general claim is that there is an overly-optimistic view of technology that states that technology can solve many, if not all, of our problems. Hence, we can enhance human beings to be better. Doubt arises, not only because of physical limitations of modifying technologies, such as in genetic engineering, drug intake and brain stimulation, but even more so because of ethical issues. Human condition is a very complex one and it has been, even if this does not seem so, analysed too little for us to drastically modify our nature. In this manner, we need an interdisciplinary and public discourse for discussions about mentioned topics - intertwining of modern values, human condition, society and techno-scientific progress.

\section{References}

[1] Max More: True Transhumanism: A reply to Don Ihde, Transhumanism and Its Critics (2010) p.138.

\begin{tabular}{llllll}
\hline 58 & Journal of Intelligent Computing Volume & 10 & Number 2 & June & 2019 \\
\hline
\end{tabular}


[2] Bostrom, N., Sandberg, A. (2009). Cognitive Enhancement - Methods, Ethics, Regulatory Challenges, Sci Eng Ethics. 15. 311-341.

[3] Husain., Metha (2011) Cognitive enhancement by drugs in health and disease. Trends in Cognitive Sciences, January 2011, 15 (1).

[4] Greely, H., et al. (2008). Towards responsible use of cognitive-enhancing drugs by the healthy. Nature, 456 (7223), 702-705.

[5] Juslian Savulescu adn Igmar Persson. Moral Enhancement, Freedom and the God Machine. Monist. 2012 July. 95 (3), 399-421

[6] Griffiths, AJ., Wessler, SR., Carroll, SB., Doebley, JF. (2015). Introduction to genetic analysis (Eleventh ed.) The Inheritance of Complex Traits, New York, NY.p.715.

[7] Simmons, D. (2008). Genetic inequality: Human genetic engineering. Nature Education 1 (1), 173.

[8] Wei, F., et al. (2001). Genetic enhancement of inflammatory pain by forebrain NR2B overexpression. Nature Neuroscience 4, 164-169.

[9] Tang, Y. P., et al. (1999). Genetic enhancement of learning and memory in mice. Nature 401, 63-69.

[10] Wezenberg, E. (2005). Modulation of memory and visuospatial processes by biperiden and rivastigmine in elderly healthy subjects. Psychopharmacology (Berl.) 181, 582-594.

[11] Chris Zarpentine: The Thorny and Ardeous Path of Moral Progress: Moral Psychology and Moral Enhancemen. Neuroethics 6. 141-153.

[12] Brem, et al. Is neuro enhancement by noninvasive brain stimulation a net zero-sum proposition? Neuroimage 2014 Jan 15; 85 (03) 1058-1068.

[13] Kapur, N. (1996). Paradoxical functional facilitation in brain behaviour research. A critical review. Brain. 119(Pt5),1775-1790. 